\title{
Les axes Nord-Sud en Haute-Saône : vers un retour de la Franche-Comté en position de carrefour
}

North-south axes in Haute-Saône: recovery of the Franche-Comté region as a crossroad

Die Nord-Süd Achsen im Departement Haute-Saône : die Region Franche-Comté auf dem Weg zurück zu einem wichtigen Verkehrsknoten

Jean-Louis Mignot

\section{OpenEdition Journals}

Édition électronique

URL : http://journals.openedition.org/rge/1521

DOI : $10.4000 /$ rge. 1521

ISSN : 2108-6478

Éditeur

Association des géographes de l'Est

Édition imprimée

Date de publication : 1 juin 2007

ISSN : 0035-3213

Référence électronique

Jean-Louis Mignot, «Les axes Nord-Sud en Haute-Saône : vers un retour de la Franche-Comté en position de carrefour », Revue Géographique de l'Est [En ligne], vol. 47 / 3 | 2007, mis en ligne le 08 novembre 2011, consulté le 07 septembre 2020. URL : http://journals.openedition.org/rge/1521 ; DOI : https://doi.org/10.4000/rge.1521

Ce document a été généré automatiquement le 7 septembre 2020

Tous droits réservés 


\title{
Les axes Nord-Sud en Haute-Saône : vers un retour de la Franche-Comté en position de carrefour
}

\author{
North-south axes in Haute-Saône: recovery of the Franche-Comté region as a \\ crossroad \\ Die Nord-Süd Achsen im Departement Haute-Saône : die Region Franche-Comté \\ auf dem Weg zurück zu einem wichtigen Verkehrsknoten
}

Jean-Louis Mignot

\section{Introduction}

Le département de la Haute-Saône a été, jusqu'au début des années 1950, un carrefour entre les axes nord-sud et les axes est-ouest. Actuellement, la Haute-Saône peut être considérée comme un espace enclavé ou plutôt déconnecté, car son territoire est contourné par toutes les grandes infrastructures de transport comme les autoroutes, les voies ferrées électrifiées, les voies fluviales à grand gabarit, alors que le reste de la Franche-Comté bénéficie d'axes structurants comme les autoroutes A36 (MulhouseBeaune), A39 (Dijon- Dôle-Bourg-en-Bresse) et les lignes ferroviaires BelfortMontbéliard-Besançon-Dijon et Strasbourg-Lyon. La Haute-Saône, quant à elle, semble être restée à l'écart de ces infrastructures de transport modernes conduisant à une déconnexion du département. Pourtant, celui-ci se situe sur le grand axe nord-sud à l'échelle européenne, entre le Benelux et l'Italie et à l'échelle française entre la Lorraine et la vallée du Rhône, faisant de la Franche-Comté un carrefour en forme de " Y ", puisque la liaison transversale Strasbourg-Lyon rejoint cet axe majeur au niveau de la vallée du Doubs. Mais cet axe majeur semble être oublié par les édiles comtois qui préfèrent se concentrer sur l'aménagement de la transversale et du Revermont ${ }^{1}$. Néanmoins, depuis la fin des années 1990, les Haut-Saônois semblent vouloir redévelopper cet axe nord-sud, car il présenterait un intérêt à l'échelle européenne, 
formant un itinéraire alternatif à l'A31 et aux lignes ferroviaires Toul-Dijon et Strasbourg-Bâle et permettrait également de relier par voie fluviale à grand gabarit le bassin de la Mer du Nord avec celui de la Mer Méditerranée. De plus, à l'échelle régionale, la liaison nord-sud assurerait une connexion du département avec Besançon et le reste de la région. Néanmoins, afin de mieux cerner la situation géographique particulière du département de la Haute-Saône, une présentation s'avère nécessaire avant d'aborder sa position au sein de la Franche- Comté et du Grand-Est. Enfin, la dernière partie sera consacrée au désenclavement du département par le renouveau de l'axe nord-sud.

\section{Les voies de communications en Haute-Saône}

\section{A. Présentation de la Haute-Saône}

2 Le département de la Haute-Saône, situé administrativement en Franche-Comté, est limitrophe des régions Lorraine, Champagne-Ardenne, Bourgogne et Alsace jusqu'en 1922, (ici avant la création du Territoire de Belfort). Il se caractérise par l'absence de grandes unités urbaines. Il compte seulement cinq villes de plus de 5000 habitants, Vesoul, Héricourt, Luxeuil-les-Bains, Lure et Gray.

Or la répartition spatiale de ces villes est originale, puisque hormis Vesoul, située au centre, toutes les autres agglomérations se localisent aux marges du département en position de contact avec les régions voisines. Ainsi Luxeuil-les-Bains, deuxième aire urbaine du département (14 145 habitants), derrière Vesoul (28 810 habitants), se situe au nord-est du département en contact avec la Lorraine, Gray (11 580 habitants) au sudest, en contact avec la Bourgogne. Lure (10 877 habitants) se place aux limites des influences lorraines et alsaciennes et Héricourt (11 027 habitants) au contact de l'Alsace et de la Franche-Comté. La position géographique des cités haut-saônoises, ainsi que la faible taille de Vesoul, font que les autres villes regardent plus vers les grandes métropoles voisines que vers Besançon. En effet, Luxeuil-les-Bains regarde plus vers Épinal et Nancy que vers Vesoul et Besançon, Gray vers Dijon, Héricourt vers Strasbourg. Lure est à la fois sous l'influence strasbourgeoise et nancéienne comme l'indique F. Braudel (1986) « En dépit des distances et des liens administratifs, c'est un fait que «Besançon ne paraît guère plus proche que Dijon à Dole ou à Gray, que Lyon à Saint-Claude, que Nancy à Luxeuil, etc. ». Ainsi Besançon, capitale régionale, ne peut pas être considérée comme le principal pôle pour le département de la Haute-Saône. Le rayonnement des grandes villes voisines sur l'espace haut-saônois s'explique par le rôle joué par les infrastructures de transport (routes, voies ferrées et voie d'eau) (cf. Figure 1 et Tableau 1). 
Figure 1 : Les infrastructures de transport en Haute-Saône

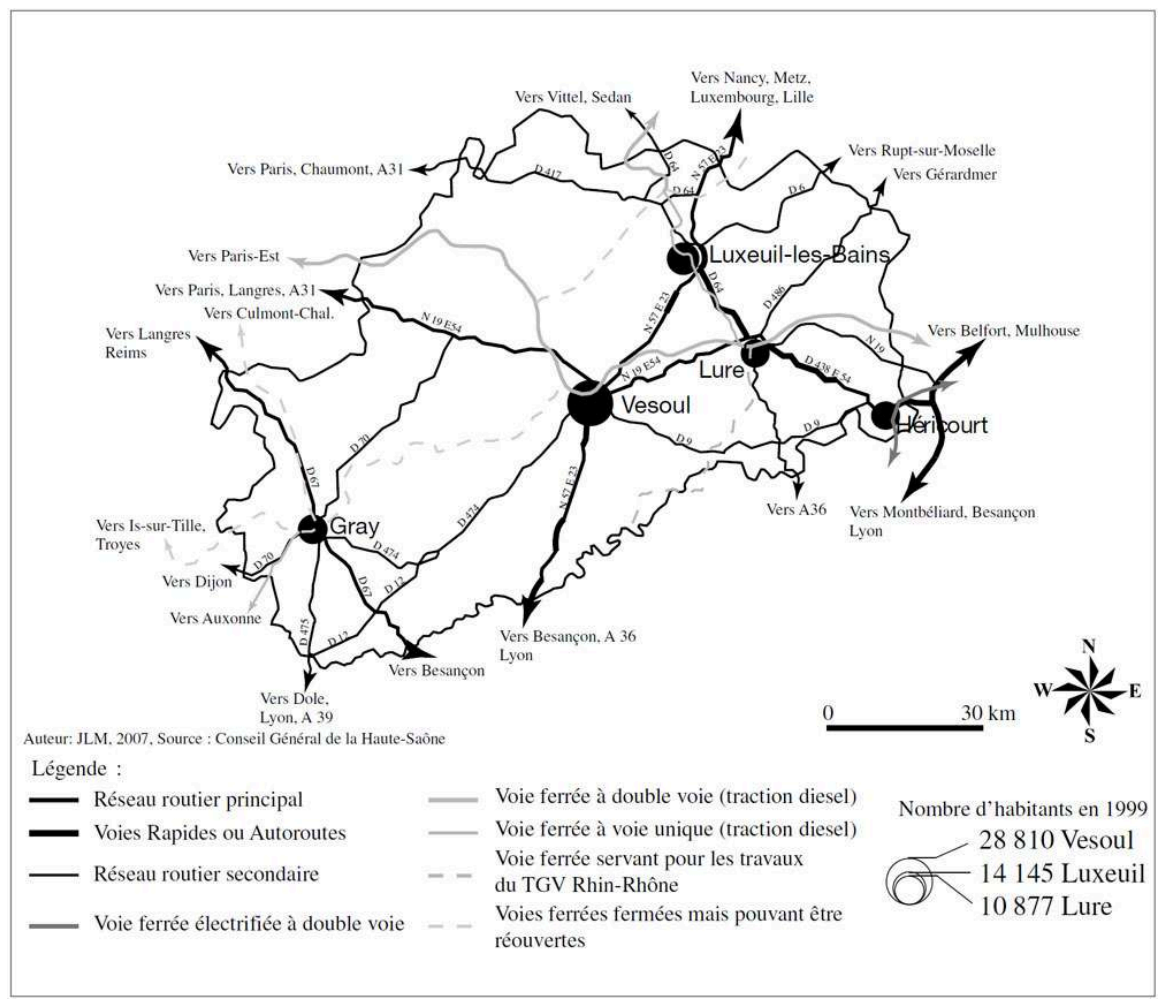

Tableau 1 : Distance-temps entre les principales villes haut-saônoises et les grandes villes de l'Est

\begin{tabular}{|c|c|c|c|c|c|c|c|c|c|c|}
\hline & \multicolumn{2}{|c|}{ Nancy } & \multicolumn{2}{|c|}{ Dijon } & \multicolumn{2}{|c|}{ Mulhouse } & \multicolumn{2}{|c|}{ Besançon } & \multicolumn{2}{|c|}{ Strasbourg } \\
\hline & $\begin{array}{l}\text { Temps de } \\
\text { parcours }\end{array}$ & $\mathrm{km}$ & $\begin{array}{l}\text { Temps de } \\
\text { parcours }\end{array}$ & $\mathrm{km}$ & $\begin{array}{l}\text { Temps de } \\
\text { parcours }\end{array}$ & $\mathrm{km}$ & $\begin{array}{l}\text { Temps de } \\
\text { parcours }\end{array}$ & $\mathrm{km}$ & $\begin{array}{l}\text { Temps de } \\
\text { parcours }\end{array}$ & $\mathrm{km}$ \\
\hline Luxeuil & 1 h 22 & 123 & $2 \mathrm{~h} 16$ & 139 & $1 \mathrm{~h} 14$ & 101 & $1 \mathrm{~h} 15$ & 87 & $2 \mathrm{~h} 11$ & 214 \\
\hline Lure & 1 h 37 & 142 & $2 \mathrm{~h} 12$ & 139 & 0 h 59 & 80 & $\begin{array}{l}1 \mathrm{~h} 11^{\star} \\
1 \mathrm{~h} 0^{\star \star}\end{array}$ & $\begin{array}{l}84 \\
82\end{array}$ & 1 h 57 & 193 \\
\hline Héricourt & 1 h 56 & 170 & $1 \mathrm{~h} 46$ & 185 & $0 \mathrm{~h} 41$ & 56 & $1 \mathrm{~h} 04$ & 101 & $1 \mathrm{~h} 39$ & 169 \\
\hline Vesoul & $1 \mathrm{~h} 50$ & 156 & 1 h 47 & 108 & $1 \mathrm{~h} 23$ & 111 & $0 \mathrm{~h} 45$ & 49 & 2 h 21 & 224 \\
\hline Gray & 2 h 23 & 197 & 0 h 48 & 49 & 1 h 55 & 178 & 0 h 48 & 54 & 2 h 53 & 291 \\
\hline
\end{tabular}

\section{B. Des flux méridiens majoritaires en Haute-Saône}

4 Après cette brève présentation du département de la Haute-Saône, une étude des flux à l'intérieur du département s'avère nécessaire, afin de comprendre le fonctionnement du territoire. Le département est traversé par des axes nord-sud et est-ouest (cf. Figure 2). Contrairement aux idées reçues, les axes principaux pour le département de la Haute-Saône sont les axes nord-sud. Ils assurent la connexion du département avec Besançon, la capitale régionale, mais surtout ils permettent de connecter le département aux espaces dynamiques (Alsace, région Bâloise, Benelux, Sillon Lorrain, Couloir Rhodanien). L'étude approfondie du trafic, tous modes confondus, montre bien cette primauté des axes nord-sud sur les axes est-ouest. Ainsi, le trafic varie du simple au double. 
Figure 2 : Les flux routiers en Haute-Saône

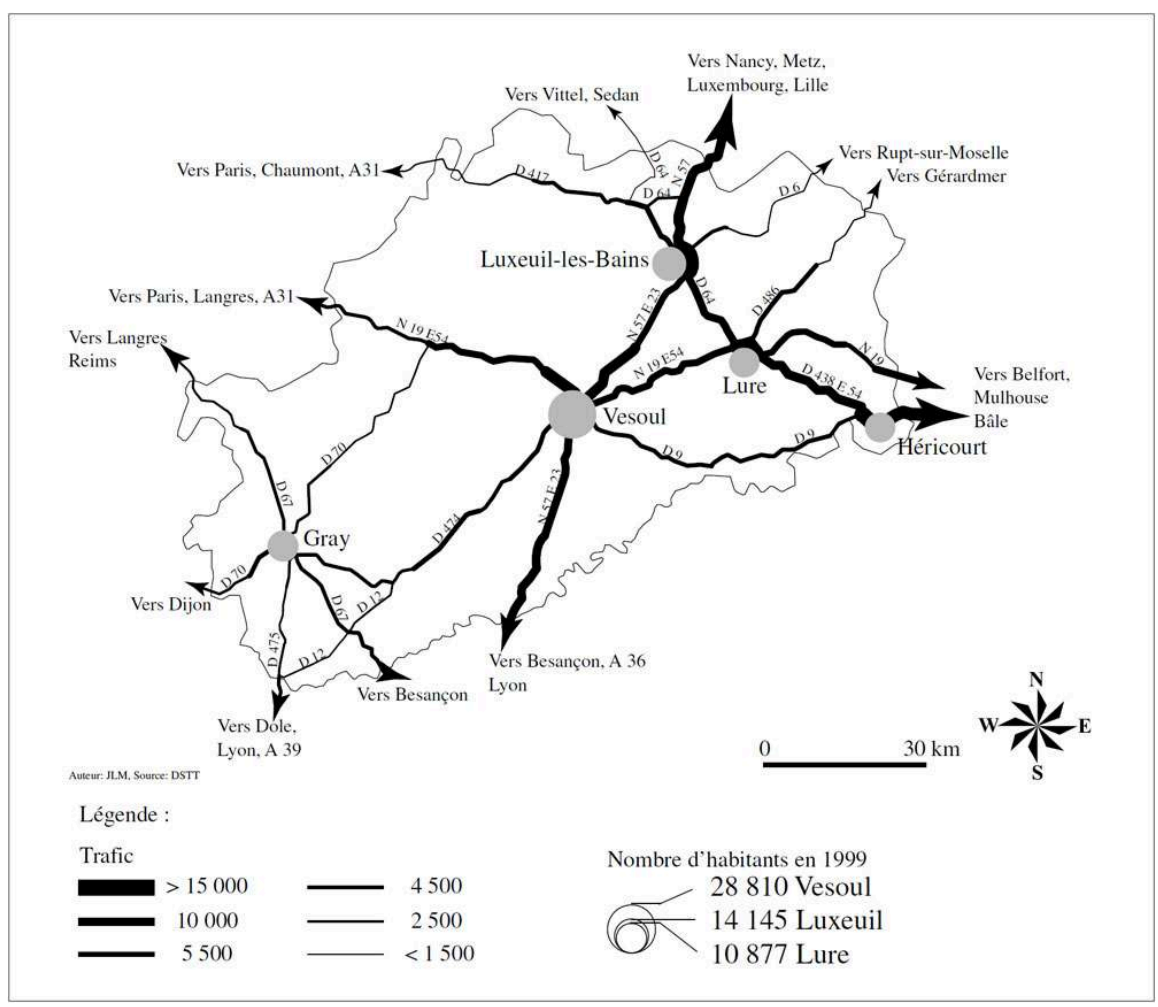

5 Les principaux axes routiers nord-sud sont la RN57/E23 qui relie à l'échelle européenne le Luxembourg à la Suisse, tandis qu'au niveau local, elle assure la liaison entre la Haute-Saône et le reste de la région et la liaison RN57-RD64-RD438 qui permet de mettre en relation le Benelux et la Lorraine avec la Suisse, tout en contournant le massif des Vosges par le Sud. Le trafic journalier sur ces deux axes varie entre 7700 et 18000 véhicules par jour (cf. Figure 2) dont 2200 à 2600 poids lourds. Toutefois, sur les différentes sections, le trafic est généralement supérieur aux 10000 véhicules par jour, notamment entre Héricourt et Luxeuil, Luxeuil et Remiremont (88) et entre Saulx-deVesoul et Besançon. Seule la section comprise entre Luxeuil et Saulx possède un trafic inférieur à 8000 véhicules par jour. Il existe également d'autres axes routiers méridiens importants, comme un itinéraire alternatif à l'A31 qui permet de rejoindre l'A39 à Dole par Luxeuil et Vesoul en empruntant la RD474, ou encore la RD67 qui permet, depuis Besançon ou Gray, d'atteindre l'A31. Le trafic est généralement aux environs de 5000 véhicules par jour, ce qui en fait des axes nettement moins importants que les précédents. Néanmoins, les axes nord-sud possèdent une qualité de service nettement inférieure à leur trafic, puisque hormis la RD64 entièrement aménagée en voie rapide, la RN57 ne compte que quelques sections modernisées, là où le trafic est le moins important, posant ainsi des problèmes d'écoulement des flux ou de dangerosité de l'axe.

6 Par rapport aux axes nord-sud, le trafic est nettement moindre sur les axes routiers longitudinaux et même sur la RN19/E54, considérée comme la route principale du département, reliant Langres (A31) à l'A36 au niveau de Belfort. Hormis aux périphéries de Vesoul et de Lure, le trafic ne dépasse pas les 8000 véhicules par jour dans le département et il n'atteint même pas le seuil des 5000 véhicules quotidiens à la limite entre la Haute-Saône et la Haute-Marne, ce qui est très faible pour une route nationale 
(cf. Figure 2). Pourtant la RN19 devrait être aménagée en autoroute entre Vesoul et Langres, alors que son trafic ne nécessite pas un tel aménagement. En plus de la RN19, le département est traversé par un autre axe est-ouest qui relie Luxeuil à l'A31, c'est la RD417 dont le trafic tourne aux environs de 3000 véhicules par jour.

7 Tout comme pour le mode routier, le principal axe fluvial utilisé est l'axe nord-sud, car la liaison est-ouest n'a pas été achevée. Le réseau fluvial nord-sud se compose du Canal des Vosges et de la Saône canalisée. Le trafic concerne essentiellement l'activité de plaisance fluviale, puisqu'il y a entre 2000 et 6000 bateaux par an qui fréquentent le canal nord-sud. Néanmoins, il subsiste une activité de fret fluvial avec un trafic qui s'établit aux environs de 100 péniches par an.

Contrairement aux deux autres modes, le mode ferroviaire se distingue par l'importance relative de l'axe est/ouest. Cette primauté s'explique aisément car la ligne est-ouest reliant Vesoul à Belfort fait partie de la ligne radiale Paris-Bâle. La relation TER Vesoul-Belfort est empruntée par 800 voyageurs par jour, mais la majorité de la clientèle effectue la liaison entre Lure et Belfort. L'axe ferroviaire nord-sud a été scindé en plusieurs lignes, une ligne qui relie Vesoul à Besançon par un service d'autocars financé par la région de Franche-Comté et qui a un trafic d'environ 600 voyageurs par jour, une ligne reliant Luxeuil à Vesoul ayant un trafic total de 70 voyageurs par jour, et enfin la liaison ferroviaire Belfort-Luxeuil-Épinal fréquentée journellement par 330 voyageurs (cf. Figure 3), dont une centaine effectuent la liaison Luxeuil-Épinal (60) ou Luxeuil-Nancy (40). Là encore, comme pour le mode routier, l'analyse du trafic révèle que les flux principaux de la ville de Luxeuil se font avec la Lorraine et non avec la Franche-Comté.

Figure 3 : Les flux de voyageurs transportés par le TER Franche-Comté

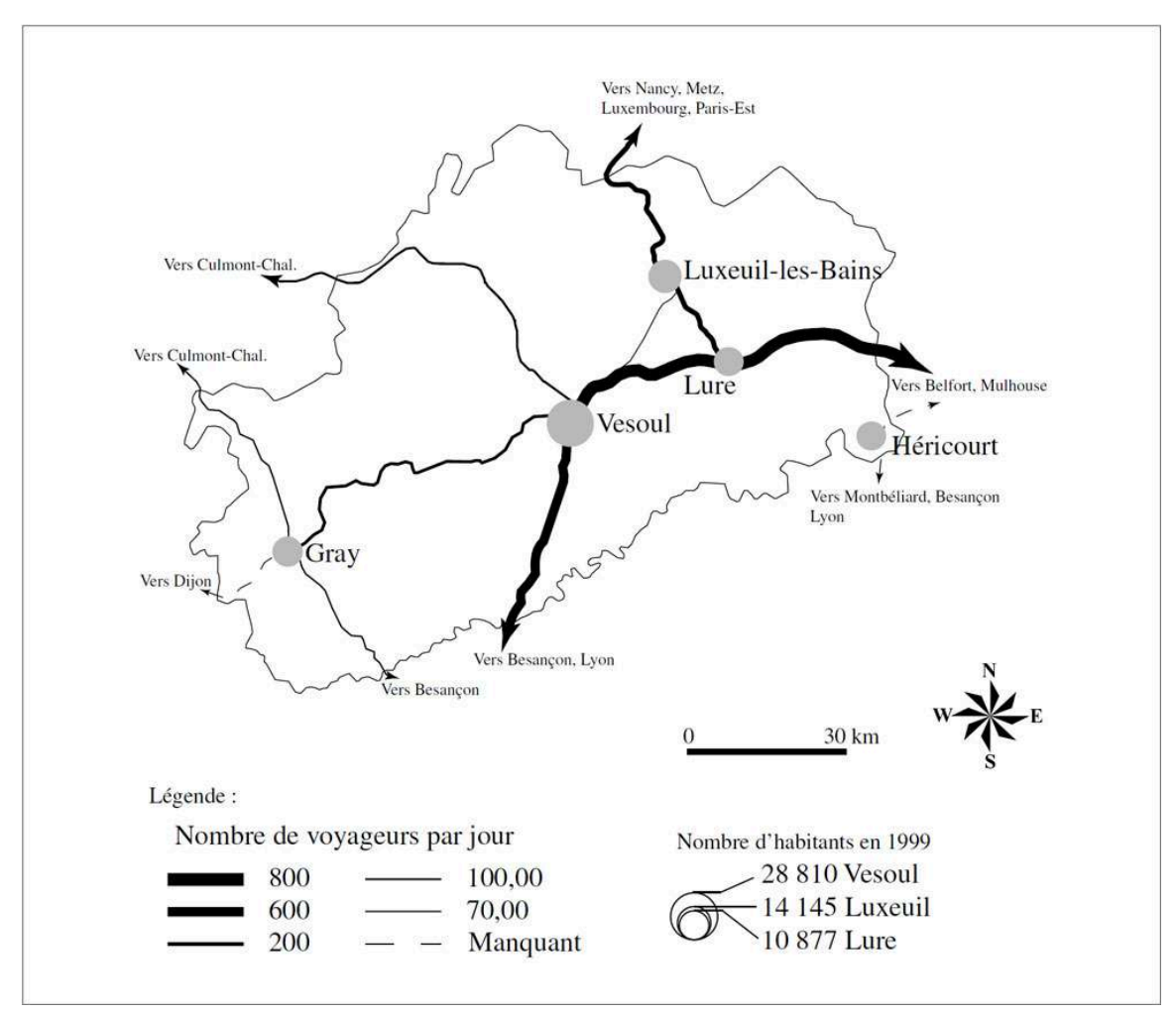


9 L'analyse des flux en fonction des différents modes de transports montre bien que les axes principaux sont les axes nord-sud et non pas les axes radiaux est-ouest. De plus, malgré leur importance, ces axes méridiens sont sous-équipés, expliquant en partie l'enclavement de la Haute-Saône. Toutefois, la solution au désenclavement du département pourrait passer par l'aménagement de ces axes majeurs, qui, par là même, désengorgeraient les principaux axes de l'Est de la France, à l'heure actuelle surchargés.

\section{Un département enclavé à l'écart des axes saturés du Grand Est}

\section{A. Un département enclavé ?}

L'enclavement est défini par J.-J. Bavoux et al. ${ }^{2}$ comme l'isolement relatif d'un lieu par rapport à des lieux proches ou de même importance, conséquence de son inaccessibilité ou de sa mauvaise accessibilité par manque d'infrastructures et/ou d'offres de transport. Cette définition fait référence aux deux notions fortes de l'accessibilité d'un lieu et de la présence d'infrastructures performantes. Or, la première partie a montré que le département de la Haute-Saône ne possédait pas de voies de communications performantes. Le département n'est desservi par aucune autoroute ou voie rapide de type autoroutier, par aucune voie ferrée électrifiée ou LGV et encore moins par une liaison fluviale à grand gabarit. Toutes ces infrastructures contournent le département ou bien s'arrêtent à la limite départementale (cf. Figure 4). L'enclavement de la HauteSaône s'explique par la politique régionale d'aménagement du territoire qui a privilégié les axes Belfort-Dijon et Besançon-Lyon, délaissant la Haute- Saône, ainsi que l'axe nord-sud Lorraine-Vallée du Rhône. Dans les années 60-70, l'État demande aux CODER ${ }^{3}$ de définir les axes prioritaires. En Franche- Comté, c'est l'axe transversal StrasbourgLyon et l'axe radial Paris-Dijon-Lausanne qui sont retenus, c'està- dire la liaison NordSud entre l'Alsace et la vallée du Rhône et la liaison radiale entre l'île de France et la Suisse Romande. Dès lors, les autoroutes A36 (Mulhouse-Beaune) et A39 (Dijon-DoleBourg-en- Bresse-Lyon) sont construites, les voies ferrées, Strasbourg-BelfortBesançon-Lons-le-Saunier-Lyon et Dijon-Dole-Pontarlier-Suisse sont électrifiées, permettant ainsi, dès 1983, aux gares de Dole, Besançon, Frasne et Pontarlier d'être desservies par le TGV. Le seul projet n'ayant pas abouti concerne la création d'un canal à grand gabarit reliant le Rhin au Rhône, car le gouvernement Jospin l'a abandonné. Les départements du Territoire de Belfort, du Doubs et du Jura possèdent donc des axes structurants et seule la Haute-Saône en demeure à l'écart. Pourtant, au début des années 70, les élus haut-saônois, notamment ceux du secteur de Luxeuil, s'intéressent à la création de l'autoroute reliant Nancy à Lyon, car la modernisation de cet axe nordsud entre la Lorraine et la vallée du Rhône permettra le désenclavement du département. Le Conseil Général demande donc l'aménagement en autoroute de la RN57, qui, déjà à cette époque-là, appartient aux GLADT ${ }^{4}$, car elle constitue "le principal axe de dégagement de la métropole Lorraine vers le Sud. $\aleph^{5}$ Or, concernant la création de l'autoroute entre Nancy et Lyon (Nancy-Beaune) deux projets sont étudiés, l'un passant par la vallée de la Meuse et Langres, donc proche de la RN74 (Chalon-surSaône-Nancy) et l'autre relatif à la création d'une autoroute proche de la RN57 desservant Épinal, Remiremont, Luxeuil, Vesoul, Besançon, Lons-le-Saunier et Lyon. 
Toutefois, il est précisé qu'en fonction du tracé retenu l'autre itinéraire fera l'objet d'un aménagement en route express, mais dans tous les cas, la Haute-Saône devrait être reliée à la Lorraine par une autoroute, afin de faciliter les échanges entre Besançon, Vesoul et Luxeuil-Saint-Loup. Ces propositions émanant de l'État et retenues par le département de la Haute-Saône ont été ignorées par la CODER de Franche-Comté, privant ainsi le département d'un axe autoroutier nord-sud et la région d'une position de carrefour entre les axes Strasbourg-Lyon et Nancy-Lyon. L'itinéraire retenu pour la construction de l'autoroute a été celui passant par la haute vallée de la Meuse. La création de l'autoroute entre Nancy et Lyon montre qu'il existe une double concurrence : l'une entre l'axe nord-sud passant par la haute vallée de la Meuse et l'axe desservant la vallée de la Moselle et la Haute- Saône et une autre à l'intérieur de la Franche-Comté entre la Haute-Saône où l'aménagement de l'axe Nancy-Lyon est primordial et le reste de la région qui regarde vers la transversale Strasbourg-Lyon.

Figure 4 : La Haute-Saône un département à l'écart des axes modernes

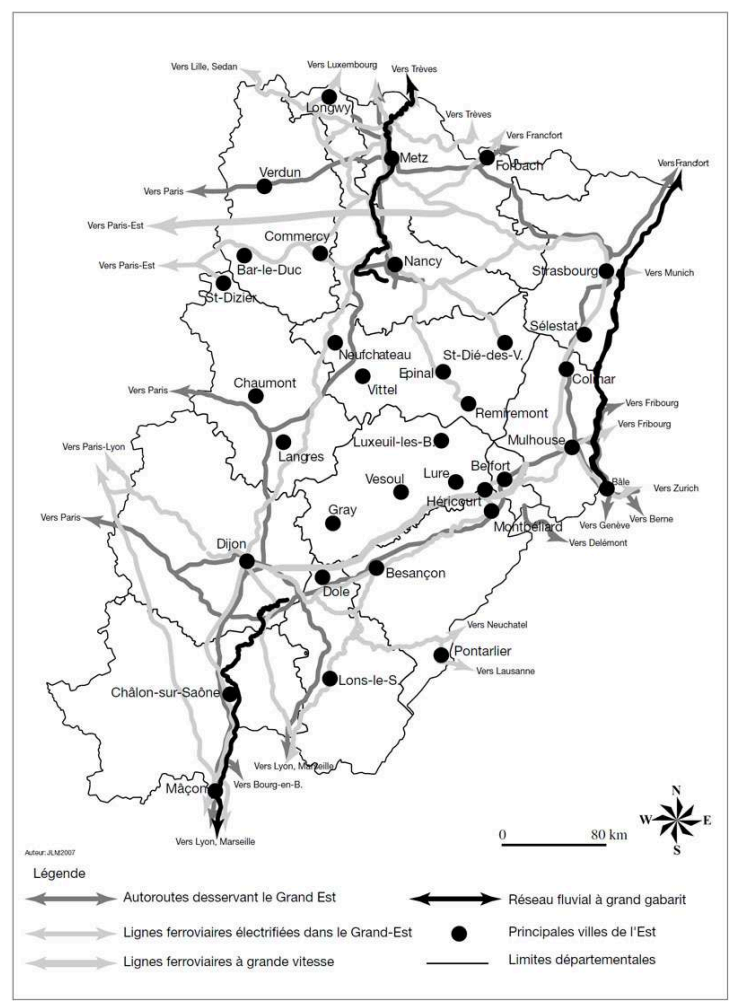

11 Or, cette double concurrence s'illustre à travers deux exemples. Tout d'abord, l'axe ferroviaire Nancy-Lyon permet de montrer la rivalité existante entre l'itinéraire suivant la vallée de la Meuse et celui passant par Épinal et la Haute-Saône. Lors de la création du chemin de fer, dans la seconde moitié du XIXe siècle, la première ligne ferroviaire nord-sud entre la Lorraine et la vallée du Rhône desservait Épinal, Aillevillers, Vesoul, Gray et Dijon. Mais la ville de Neufchâteau, s'estimant lésée, réclama elle aussi une liaison ferroviaire avec Dijon, qu'elle obtint à la fin du XIXe siècle, l'activité principale de cette ligne devant être le transport de troupes en temps de guerre et non l'activité de voyageurs.

12 Or, en 1960, la décision d'électrifier la ligne via Neufchâteau a été prise, délaissant ainsi la ligne historique nord-sud via Épinal et Aillevillers. La répercussion de cette décision 
a été longue à venir, puisque c'est seulement, en 1991, avec la fermeture de la ligne Aillevillers Port d'Atelier qu'Épinal et la Haute-Saône ont perdu la liaison ferroviaire Nancy-Lyon, réduisant donc l'accessibilité ferroviaire de la Haute-Saône et d'Épinal. Cet exemple illustre bien la concurrence entre les deux axes nord-sud.

Le deuxième exemple est relatif à l'aménagement de la liaison fluviale à grand gabarit entre la Mer du Nord et la Mer Méditerranée. Au début des années 1950, il est envisagé de créer une liaison fluviale à grand gabarit entre la Mer du Nord et la Méditerranée ayant un tronc commun jusqu'à Saint-Symphorien (21), puis deux branches, l'une se dirigeant vers la Lorraine par la vallée de la Saône, défendue par la Haute-Saône et la Lorraine, abandonnée au milieu des années 80 et l'autre vers l'Alsace par la vallée du Doubs soutenue par la CODER de Franche-Comté et l'Alsace, abandonnée en 1997. Or l'abandon du projet de canal Rhin-Rhône a permis de relancer l'idée d'une liaison Saône-Moselle à grand gabarit portée par les régions Lorraine et Rhône- Alpes, et suivie de près par la Haute-Saône. Mais l'émergence de la liaison Saône-Moselle, a fait naître en Alsace et dans le reste de la Franche-Comté, l'idée d'une liaison Saône-Rhin, également à grand gabarit via la vallée du Doubs. Or sur ces projets, il existe une concurrence entre la Haute-Saône et le reste de la Franche-Comté.

L'enclavement de la Haute-Saône résulte donc d'une part de la concurrence entre les deux axes nord-sud reliant la Lorraine à la vallée du Rhône et d'autre part de la concurrence existant entre le département de la Haute-Saône et le reste de la FrancheComté. Cette situation d'enclavement, place le département en position d'angle mort par rapport aux axes structurants du Grand-Est. Toutefois, ces axes connaissent actuellement des problèmes de saturation et ils constituent dès lors une entrave aux échanges.

\section{B. Problèmes et enjeux territoriaux dans le Grand-Est}

15 Les axes structurants du Grand-Est connaissent des problèmes pour écouler les flux ${ }^{6}$ ( $\mathrm{cf}$. Figure 5). Les problèmes sont de deux sortes, soit l'axe arrive à saturation, comme en Lorraine (A31, voie ferrée Toul- Dijon) ou en Alsace (A35 ou voie ferrée MulhouseBâle), soit l'axe possède un gabarit inadapté ou un profil difficile, ce qui entrave les échanges nord-sud, comme par exemple les lignes ferroviaires Belfort- Besançon et Toul-Dijon, ou encore l'autoroute A36 qui comporte de nombreuses sections limitées à $110 \mathrm{~km} / \mathrm{h}$. Hormis les problèmes rencontrés sur les différents axes, certains nœuds arrivent à saturation, tels que Metz, Bâle ou Dijon. D'autres gares, comme celle de Nancy, sont trop petites pour le trafic ferroviaire qu'elles reçoivent (TER, fret, TGV, Corail). De plus, les lignes TGV Est et Rhin-Rhône qui desserviront à terme l'Est de la France contourneront également la Haute- Saône, sans desservir le territoire départemental. Certes, le TGV n'a pas vocation à desservir tous les territoires traversés, contribuant parfois plus à enclaver certains espaces, comme par exemple la ville de Vesoul. Toutefois, le TGV-Est et le TGV Rhin-Rhône ont des missions totalement différentes. La principale mission du TGV-Est européen est d'assurer les liaisons entre l'Est de la France, la région parisienne et les régions de l'Ouest, du Sud-Ouest et du Nord de la France. Alors que le TGV Rhin-Rhône a pour rôle de connecter l'Est au Sud-Est de la France et dans le cadre européen l'Allemagne à l'Espagne, formant donc un TGV transversal. Néanmoins, le TGV Rhin-Rhône desservira directement l'Alsace, la Franche-Comté, la Bourgogne et Rhône-Alpes, si bien que la Lorraine et le Luxembourg resteront à l'écart de cette liaison nord-sud à grande-vitesse. Dès lors, se pose le 
problème des liaisons directes nord-sud entre la Lorraine et le Sud-Est de la France, car actuellement la Lorraine est desservie par des trains Luxembourg/Nice ou Metz/ Grenoble et Metz/Lyon. Toutefois, ces dessertes sont à relativiser, puisque seules les gares de Metz et de Nancy sont desservies par tous les trains nord-sud. La gare de Neufchâteau, quant à elle, était également desservie, depuis 1991, par tous les trains nord-sud, suite à la suppression de la desserte transversale méridienne par Épinal. Mais, après la mise en service du TGV Metz/Nice, la desserte de la gare vosgienne a été supprimée par manque de voyageurs. En effet, face à la volonté politique de la Lorraine, la SNCF a testé l'arrêt de ce TGV en gare de Neufchâteau pendant un an. Finalement, l'arrêt à été définitivement supprimé vu le faible nombre de personnes empruntant le train. De plus, la ligne ferroviaire nord-sud Toul-Neufchâteau-Dijon, pose plusieurs problèmes. Elle est saturée à certaines périodes de la journée, son profil ne permet pas une vitesse commerciale élevée pour les trains de voyageurs et elle ne dessert pas des zones densément peuplées. Dès lors, l'arrivée du TGV Rhin-Rhône est l'occasion de réfléchir sur la revitalisation de l'axe ferroviaire nord-sud par Épinal et la Haute-Saône. En plus de la liaison ferroviaire nord-sud, la ville de Neufchâteau bénéficie également de l'autoroute A31, mais cette infrastructure n'apporte quasiment aucun développement à la cité vosgienne et de la même manière la ville d'Épinal et la HauteSaône sont contournées par cette infrastructure.

Figure 5 : Un espace enclavé encadré par des axes saturés

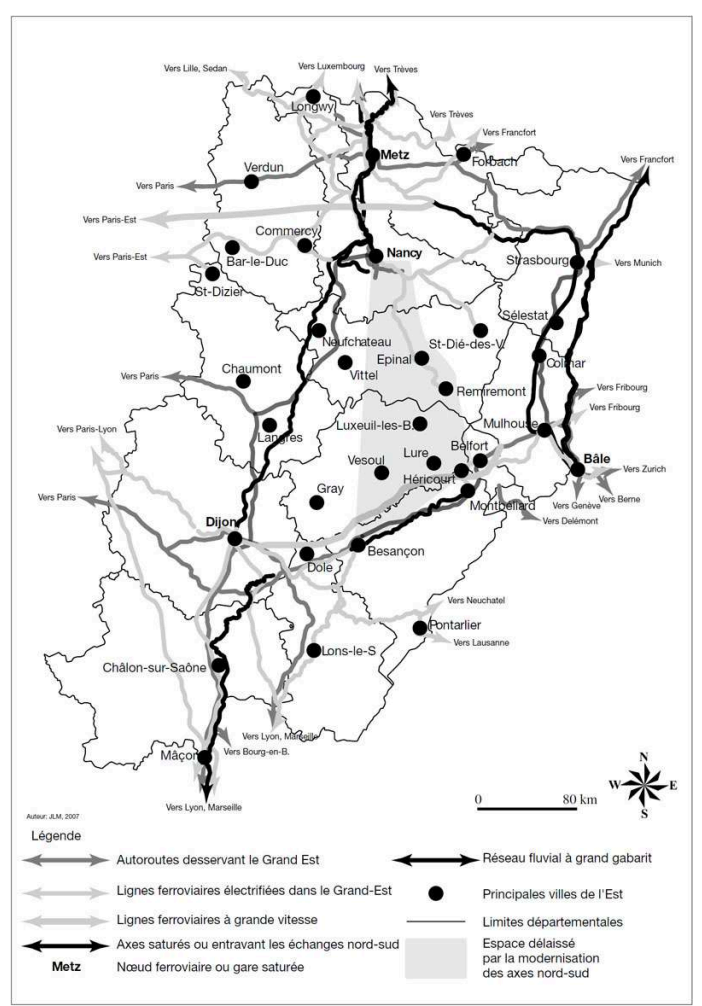

16 Les axes nord-sud en Lorraine, décrits précédemment, connaissent donc des problèmes (cf. Figure 5) et délaissent un espace plus densément peuplé, situé plus à l'Est, formé par la ville d'Épinal et la Haute-Saône.

17 Dès lors, l'arrivée du TGV Rhin-Rhône, les problèmes de saturation rencontrés sur l'A31 entre Luxembourg et Toul, ou encore le projet de liaison fluviale nord-sud à grand 
gabarit Saône-Moselle, sont peut être l'occasion de revitaliser cet axe nord-sud oublié par les grands aménagements des années 1960 (cf. Figure 5).

\section{Le renouveau des axes nord-sud : vers un désenclavement de la Haute-Saône et le retour du carrefour franc-comtois} peut se retrouver dans ce cas. En décembre 2011, sera mis en service le TGV RhinRhône, reliant dans le contexte national Strasbourg à Lyon, mais dans le même temps reste posé le problème de l'amélioration des liaisons méridiennes entre la Lorraine et la vallée du Rhône. Or à l'horizon 2012, plusieurs scénarii peuvent être envisagés. Le premier consiste à faire circuler des TGV entre Metz et Nice par l'itinéraire actuel, mais celui-ci n'est pas très performant en distance-temps et les territoires situés entre Nancy et Dijon seraient oubliés. La seconde solution serait de créer une liaison TGV entre Metz et Nice empruntant la LGV Est européenne puis l'interconnexion en Île de France, afin de rejoindre les LGV Paris Sud Est et Méditerranée, mais cette hypothèse présente l'inconvénient d'allonger la distance kilométrique et un coût élevé pour l'usager, même si le TGV a une tarification de marché. Ces deux premières solutions ne paraissent pas très réalistes. Toutefois, il reste encore à étudier deux hypothèses plus envisageables. La première serait de faire circuler des TGV entre Luxembourg et Strasbourg desservant la gare de Metz. Cette solution permettrait aux Lorrains de rejoindre le TGV Rhin-Rhône en gare de Strasbourg. Elle est avantageuse pour la Lorraine du Nord, car cet espace profiterait de la seconde phase de la LGV Est qui permettra d'effectuer la liaison Metz-Strasbourg en vingt trois minutes au lieu d'une heure vingt actuellement. Une telle liaison permet de gagner une heure sur le temps de parcours entre Metz et Marseille. Toutefois, il existe deux contraintes, la première est conditionnée à la réalisation de la deuxième phase de la LGV Est et la deuxième concerne particulièrement le domaine de l'aménagement du territoire, car une telle solution enclave à terme tout le Sud de la Lorraine. 
21 La deuxième hypothèse consiste à faire circuler des TGV entre le Luxembourg et le Sud de la France par la ligne ferroviaire Metz-Nancy-Épinal-Belfort. Ainsi les TGV entre la Lorraine et le Sud de la France rejoindraient la LGV Rhin-Rhône en Franche-Comté. Cette solution présente plusieurs avantages, car, d'une part, elle offre des temps de parcours corrects depuis les villes lorraines pour rejoindre Lyon ou Marseille (cf. Tableau 2) et d'autre part, elle permet d'envisager à terme des liaisons rapides entre les différentes villes du Sillon Lorrain et le Nord de la Franche-Comté. Un autre avantage se situe dans la desserte du territoire, puisqu'une telle relation permet de desservir directement les principales villes de la Lorraine (Metz, Nancy et Épinal) et de leur offrir un débouché vers le Sud de la France plus performant qu'actuellement. De plus, la création d'une liaison permettrait de consolider les relations entre le Nord de la Franche-Comté et la Lorraine comme le préconise la DIACT ${ }^{7}$. À l'horizon 2020, la DIACT recommande que " la notion trop étroite de métropole lorraine Nancy-Metz soit dépassée par l'émergence d'un réseau de villes du sillon Mosellan allant de Thionville à Épinal, ouvre la perspective d'un renforcement des liens avec le Luxembourg, la province de Trèves et la Sarre, équilibré par une ouverture de Nancy vers le Sud-Est audelà d'Épinal (Nord Franc-Comtois) ». Or, si la première partie de la recommandation existe déjà par la création de la grande région Sarre-Lor-Lux, la seconde partie de l'avis de la DIACT est loin d'être réalisée, malgré le fait qu'il existe de fortes relations entre la Lorraine et la Haute-Saône (cf. première partie). Dès lors, une coopération doit être mise en place entre la Lorraine et le Nord de la Franche-Comté (Haute-Saône et Territoire de Belfort), qui à terme pourra déboucher sur la création d'un réseau de villes allant de Luxembourg à Belfort-Montbéliard et intégrant des villes comme Remiremont, Luxeuil et Lure.

Tableau 2 : Distance-temps entre les différentes relations nord-sud méridiennes

\begin{tabular}{|c|c|c|c|c|c|c|c|c|}
\hline \multirow[b]{2}{*}{ Parcours } & \multicolumn{3}{|c|}{ Actuellement } & \multicolumn{3}{|c|}{$\begin{array}{l}\text { TGV Rhin-Rhône via } \\
\text { la Haute-Saône }\end{array}$} & \multicolumn{2}{|c|}{$\begin{array}{l}\text { TGV Rhin-Rhône } \\
\text { via Strasbourg }\end{array}$} \\
\hline & $\begin{array}{l}\text { via Nancy, } \\
\text { Neuf- } \\
\text { château }\end{array}$ & $\begin{array}{l}\text { via } \\
\text { Belfort }\end{array}$ & $\begin{array}{c}\text { via } \\
\text { Ule-de- } \\
\text { France } \\
\text { (Tgv Est) }\end{array}$ & $\begin{array}{c}\text { Branche } \\
\text { Est }\end{array}$ & $\begin{array}{c}\text { Branches } \\
\text { Est et } \\
\text { Sud }\end{array}$ & $\begin{array}{c}\text { TGV } \\
\text { Rhin-Rhône avec } \\
\text { raccordement } \\
\text { en Franche-Comté } \\
\text { et amélioration } \\
\text { de la ligne } \\
\text { Epinal-Belfort }\end{array}$ & $\begin{array}{c}\text { Branche } \\
\text { Est }\end{array}$ & $\begin{array}{c}\text { Branches } \\
\text { Est et } \\
\text { Sud }\end{array}$ \\
\hline Epinal-Lyon & $\begin{array}{c}5 \mathrm{~h} 12 \\
(6 \mathrm{~h} 12)\end{array}$ & $4 \mathrm{~h} 55$ & 5 h 30 & 3 h 30 & $2 \mathrm{~h} 20$ & $\begin{array}{c}2 \text { h } 55 \\
(1 \text { h } 45)\end{array}$ & 5 h 50 & $4 \mathrm{~h} 40$ \\
\hline Epinal-Marseille & $\begin{array}{c}6 \text { h h } 56 \\
(7 \text { h } 56)\end{array}$ & 7 h 03 & 7 h 20 & 5 h 30 & 3 h 20 & $\begin{array}{c}4 \text { h } 55 \\
(2 \text { h } 45)\end{array}$ & $7 \mathrm{~h} 10$ & $6 \mathrm{~h} 00$ \\
\hline Nancy-Lyon & $\begin{array}{c}4 \text { h } 35 \\
(5 \text { h } 35)\end{array}$ & 5 h 40 & 4 h 45 & 4 h 25 & 3 h 15 & $\begin{array}{l}3 \text { h } 50 \\
(2 \text { h } 30)\end{array}$ & 4 h 35 & 3 h 25 \\
\hline Nancy-Marseille & $\begin{array}{c}6 \mathbf{6} \text { h } 12 \\
(7 \text { h } 12)\end{array}$ & 7 h 48 & 6 h 35 & $6 \mathrm{~h} 00$ & $4 \mathrm{~h} 30$ & $\begin{array}{c}5 \text { h } 25 \\
(3 \text { h } 55)\end{array}$ & $6 \mathrm{~h} 10$ & 5 h 00 \\
\hline Metz-Lyon & $4 \mathrm{~h} 46$ & $\begin{array}{l}7 \text { h } 27 \\
(6 \text { h } 02)\end{array}$ & 5 h 00 & $5 \mathrm{~h} 20$ & $4 \mathrm{~h} 00$ & $\begin{array}{c}4 \text { h } 05 \\
(3 \text { h 25) }\end{array}$ & $4 \mathrm{~h} 40$ & 3 h 25 \\
\hline Metz-Marseille & 6 h 29 & $9 \mathrm{~h} 45$ & 5 h 35 & 7 h 15 & 5 h 45 & $\begin{array}{c}5 \text { h h } 40 \\
(5 \text { h } 10)\end{array}$ & $6 \mathrm{~h} 10$ & $5 \mathrm{~h} 00$ \\
\hline
\end{tabular}

La création d'une telle liaison nord-sud entre la Lorraine et la Franche-Comté permettrait d'aboutir à cette coopération (cf. Figure 6). Néanmoins, l'amélioration de la liaison ferroviaire nécessite des investissements dans l'infrastructure elle-même, afin d'améliorer la performance. Tout d'abord, il est impératif d'électrifier la section entre Épinal et Belfort, afin de permettre aux TGV de circuler sur cette ligne. L'amélioration de cette ligne ferroviaire et notamment sa mise à $200 \mathrm{~km} / \mathrm{h}$ permettrait d'offrir un axe 
alternatif à la ligne Strasbourg-Mulhouse. Cette modernisation est réalisable et doit donc être considérée comme un objectif à moyen terme. En outre, il faut prévoir un raccordement depuis la LGV Rhin-Rhône permettant aux trains venant de Lorraine de pouvoir accéder à la ligne à grande vitesse. Une telle liaison permettrait donc à la Lorraine de bénéficier de relations rapides vers le Sud de la France, à la Haute-Saône de se désenclaver par son repositionnement sur un axe nord-sud, et à la Franche-Comté de se placer sur un nœud TGV. De plus, la création d'une telle liaison ne servirait pas qu'aux flux nord-sud, car la modernisation de la ligne Épinal-Belfort permettrait des liaisons TGV entre la Haute-Saône et l'̂̂le de France via la LGV-Est européen.

Figure 6 : Des solutions aux liaisons nord-sud à grande vitesse

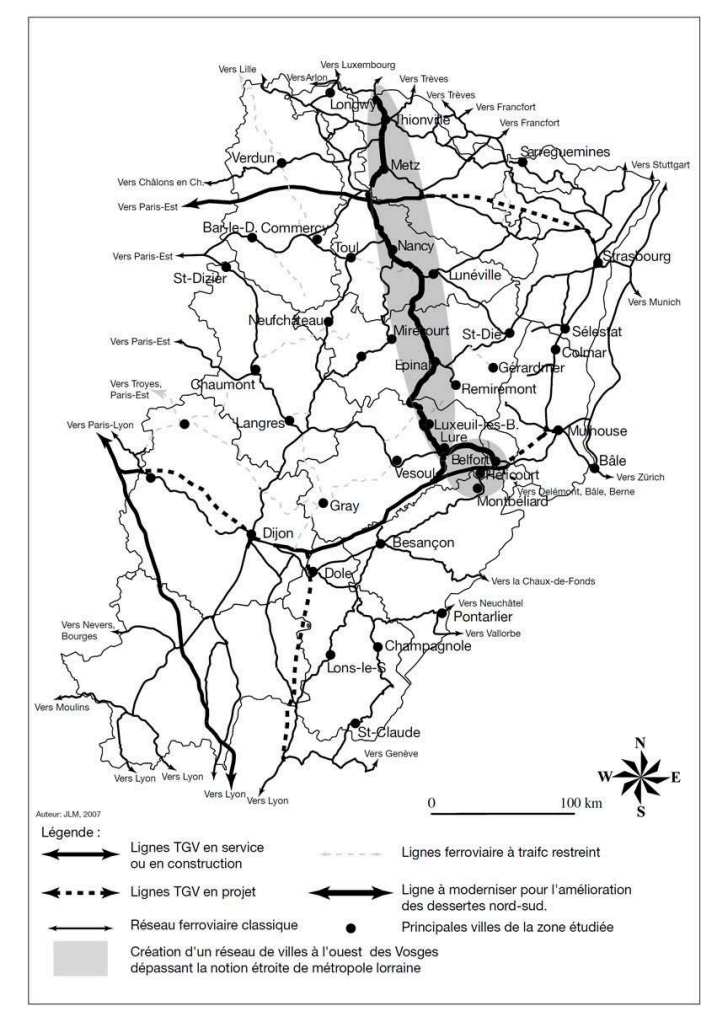

Jusqu'au 10 juin 2007, la Haute-Saône était desservie par la ligne internationale ParisMulhouse-Bâle, mais avec la mise en service du TGV-Est, la desserte entre le Grand-Est de la France et l'île de France a été refondée. Désormais, les trains à destination de la Suisse alémanique empruntent la LGV Est et passent par Strasbourg réduisant un peu plus l'accessibilité de la Haute-Saône. Or, la ligne nord-sud, outre le fait qu'elle mettrait en communication la Lorraine avec le TGV Rhin-Rhône, présente l'avantage de relier le nord de la Franche-Comté à la LGV-Est. Ainsi, cet espace nord franc-comtois, notamment le nord-est de la Haute-Saône, qui profite déjà du TGV-Est par une amélioration des temps de parcours, puisque le trajet entre Luxeuil-les-Bains et ParisEst s'effectue en $3 \mathrm{~h} 10 \mathrm{au}$ lieu de $4 \mathrm{~h} 20$, pourrait à moyen terme être relié directement à Paris. De plus, ce temps de parcours pourrait être également réduit de 30 minutes en évitant les changements en gare de Nancy et d'Épinal.

La revitalisation de la ligne ferroviaire nord-sud (Nancy-Épinal-Belfort) revêt donc un triple intérêt, car il permet d'ouvrir la Lorraine vers le sud de la France, de repositionner la Haute-Saône sur un grand axe ferroviaire Nord-Sud entre le Benelux et 
l'Espagne, et d'offrir aux villes de la Haute-Saône une liaison rapide avec Paris et par conséquent une concentration des flux nord-sud et est-ouest.

\section{Conclusion}

La situation d'enclavement de la Haute-Saône résulte d'un délaissement depuis les années 50 de l'axe nord-sud. Or, actuellement, les problèmes d'écoulement des flux méridiens entre la Lorraine et le Sud- Est font que de nouvelles solutions doivent être envisagées, comme le montre l'exemple du ferroviaire. De plus, le Grand Est constitue une impasse pour la voie d'eau à grand gabarit, puisque le canal venant de la Mer du Nord s'arrête à Neuves Maisons (54) et ne continue pas vers le Sud en direction de la Saône. Cette rivière n'est canalisée que jusqu'à St Symphorien (21) et n'est pas prolongée vers le Nord. Là encore, cet exemple aurait pu être développé, car le maillon manquant peut également passer par la Haute-Saône, participant à son désenclavement. Néanmoins, malgré la déchéance de l'axe nord-sud entre la Lorraine et la vallée du Rhône via la Franche- Comté, les principaux axes haut-saônois restent orientés de façon méridienne, comme l'ont démontré les trafics sur la RN57 et sur la RD64. Les enjeux principaux restent le désenclavement de la Haute-Saône qui se repositionnerait sur un grand axe nord-sud et permettrait à la Franche-Comté de retrouver une place de carrefour entre les deux principaux axes nord-sud de l'Est, à savoir Lorraine-vallée du Rhône et Alsacevallée du Rhône et par conséquent la région ne serait plus une région de transit, mais une région carrefour. Ce repositionnement pourrait se faire à l'occasion de l'arrivée du TGV Rhin-Rhône et de la création du canal à grand gabarit Saône-Moselle. Mais il faut que les Franc-Comtois s'intéressent d'avantage à cet axe et que les aménageurs ne considèrent plus Neufchâteau comme le principal foyer de peuplement entre Nancy et Dijon, alors que des espaces plus densément peuplés se situent dans la vallée de la Moselle, prolongés au sud par les villes de la Haute-Saône.

\section{BIBLIOGRAPHIE}

Aménager la France en 2020 (2002). - Le Grand-Est, La Documentation Française, Paris, 155 p.

AUPHAN E. (1991). - Quel avenir pour les réseaux ferrés d'Europe occidentale ?, CNRS, Paris, 202 p.

BAVOUX J.-J., BEAUCIRE F., CHAPELON L., PIERRE Z. (2005). - Géographie des transports, Armand Colin, Paris, 231 pages.

D.A.T.A.R. (2003). - La France en Europe : Quelle ambition pour la politique des transports ?, La Documentation Française, Paris, 310 p.

MIGNOT J.-L. (2005). - L'arrivée de la grande vitesse ferroviaire une solution au désenclavement de la Haute-Saône et aux problèmes de l'axe nord-sud, Cahiers scientifiques du transport, $\mathrm{n}^{\circ}$ 48/2005, p. 15-43. 
PLASSARD F. (2003). - Transport et Territoire, Prédit, La Documentation Française, Paris, 97 p.

TROIN J.-F. (1995). - Rail et Aménagement du territoire. Des héritages aux nouveaux défis, Edisud, Aixen-Provence, $264 \mathrm{p}$.

TROIN J.-F. (1997). - Les gares T.G.V. et le territoire : débat et enjeux, Annales de Géographie, ${ }^{\circ}$ 593-594, p. 34-50.

\section{NOTES}

1. Revermont : ce nom désigne l'espace géographique situé au pied du massif jurassien compris entre la ville de Lons-le-Saunier et celle de Bourg-en-Bresse.

2. Bavoux J.J., Beaucire F., Chapelon L., Zembri P., (2005), Géographie des transports, Armand Colin, Paris, 231 pages.

3. CODER : Commission de Développement Économique Régional.

4. GLADT : Grandes Liaisons d'Aménagement du Territoire.

5. Délibération du CG 70, ADHS.

6. J.-L. Mignot (2005), L'arrivée de la grande vitesse ferroviaire, une solution au désenclavement de la Haute-Saône et aux problèmes de l'axe nord-sud, Cahiers scientifiques du transport, $n^{\circ} 48$, p. 15-43.

7. DIACT : Délégation Interministérielle à l'Aménagement et la Compétitivité des Territoires

8. Nord de la Franche-Comté : expression utilisée pour désigner l'espace géographique du Nord et du Nord-Est de la Haute-Saône, du Territoire de Belfort et du Pays de Montbéliard.

\section{RÉSUMÉS}

Le département de la Haute-Saône est traversé par l'axe nord-sud reliant la Lorraine à la vallée du Rhône. Cette voie de communication a été délaissée depuis le milieu des années 1960, entrainant l'enclavement du département par certains modes de transport. Or actuellement, cet axe abandonné retrouve un intérêt croissant, car il assure le débouché vers le Sud de la région Lorraine et du Benelux. Des projets d'aménagement sont à l'étude comme l'électrification de la ligne entre Épinal et Belfort, afin d'assurer une connexion ferroviaire entre la Lorraine et le TGV Rhin-Rhône, ou encore l'étude de la liaison fluviale entre la Saône et la Moselle. Ces aménagements pourront peut-être à terme désenclaver la Haute-Saône et redonner à la FrancheComté sa position de carrefour.

The department of Haute-Saone is traversed by the north south axis connecting Lorraine to the Rhone Valley. This line of communication has been neglected since the mid-1960s, but is generating more interest because it ensures the southern connection between Lorraine and Benelux and the Rhone Valley. Some planning projects are under consideration such as electrification of the Épinal-Belfort railway line, so as to ensure a connection between the Saone and Moselle rivers. These planning projects could eventually open up the Haute-Saone department and resurrect the Franche-Comté as a crossroad. 
Die Nord-Süd Verkehrsachsen, welche Lothringen und das Rhônetal miteinander verbinden, verlaufen durch das Departement Haute-Saône. Seit der Mitte der 60er Jahren verloren diese an Bedeutung, und das Departement Haute-Saône geriet in eine periphere Lage. Neuerdings finden diese vernachlässigten Achsen wieder ein steigendes Interesse, da sie die Verbindung nach Südlothringen und die Benelux-Staaten gewährleisten. So werden derzeit verschiedene Raumordnungsprojekte geprüft, wie die Elektrifizierung der Eisenbahnlinie Epinal-Belfort, welche eine Verbindung mit Lothringen und dem TGV Rhein- Rhône ermöglicht oder das Projekt einer Wasserstraßenverbindung zwischen der Saône und der Mosel. Durch diese Projekte könnte die Abkopplung der Haute-Saône beendet und die Knotenpunktfunktion der Franche-Comté wieder hergestellt werden.

\section{INDEX}

Schlüsselwörter : Franche-Comté, Haute-Saône, Nord-Süd Achse, Raumordnungplan, Rhonetal, TGV Linie, Wasserstraßenverbindung

Mots-clés : aménagement, axe nord-sud, Franche-Comté, Haute-Saône, liaison fluviale, Lorraine, TGV, Vallée du Rhône

Keywords : Franche-Comté, Haute-Saône, land development, Lorraine, north-south axis, Rhone Valley, TGV lines, waterway

\section{AUTEUR}

\section{JEAN-LOUIS MIGNOT}

CERPA - Université de Nancy 2 - 23, bd Albert 1er54015 Nancy Cedex 